\title{
Estudio bibliométrico de la producción científica de la Revista de Nutrição a través de la Red SciELO (2001 a 2007)
}

\author{
Bibliometric study of the scientific production \\ of the Revista de Nutrição through the \\ SciELO network (2001 to 2007)
}

Vicente TOMÁS-CASTERÁ ${ }^{1}$

Javier SANZ-VALERO'

Carmina WANDEN-BERGHE ${ }^{2}$

RE S U M E N

\section{Objetivo}

Evaluar, mediante el análisis bibliométrico, la actividad científica, la producción y el consumo de información de la Revista de Nutrição, como publicación científica del área de las ciencias de la salud, en el periodo 2001 a 2007.

\section{Metodos}

Estudio descriptivo trasversal de la actividad y la producción de información científica publicada en la Revista de Nutrição, a través de la Red Scientific Electronic Library Online, en el periodo estudiado.

\section{Resultuado}

Se publicaron un total de 368 artículos. El número de artículos originales fue de 241 (65,49\%), con un índice de productividad del 2,38. Se identificaron 85 instituciones con trabajos publicados en la revista. El idioma predominante es el portugués, con 349 artículos (94,84\%). Se contabilizaron 1574 palabras clave, de las cuales 1135 (72,11\%) correspondían a los términos DeCS o MeSH;102 artículos (27,72\%) presentaban todas las palabras clave equivalentes a Descriptores. El estudio de las palabras clave muestra una orientación claramente comunitaria. La obsolescencia de las referencias citadas en Revista de Nutrição, medida por la Mediana es de 11 y el Índice de Price es del 11,69\%. El porcentaje de autocitas es de 2,08\%.

\section{Conclusione}

En términos generales la Revista de Nutrição, publicación de ámbito internacional, se mantiene dentro de los indicadores bibliométricos de las revistas de las ciencias de la salud publicadas en países iberoamericanos.

\footnotetext{
${ }^{1}$ Universidad de Alicante, Departamento de Enfermería Comunitaria, Medicina Preventiva y Salud Pública e Historia de la Ciência. Campus de Sant Vicent del Raspeig. Ap. Correos 99, E 03080, Alicante, España. Correspondencia para/Correspondence to: J. SANZ-VALERO. E-mail: <javier.sanz@ua.es>.

2 Universidad Cardenal Herrera CÉU. Elche, España.
} 
792 | V. TOMÁS-CASTERÁ et al.

Presenta un porcentaje excelente de equivalencia entre las Palabras Clave y los Descriptores de Ciencias de la Salud. La obsolescencia de sus referencias es algo elevada, situación que fue corregida en los años 2006 y 2007, los últimos del estudio.

Palabras clave: Acceso a la información. Artículo de revista. Bibliometria. Indicadores bibliométricos. Medical subject headings.

\section{A B S T R A C T}

\section{Objetive}

This study made a bibliometric analysis of the scientific production and information production and consumption of the Revista de Nutrição as a scientific journal in the field of the health sciences, between 2001 and 2007.

\section{Methods}

This is a descriptive, cross-sectional study on the scientific activity and information production published by the Revista de Nutrição, through the SciELO network in the abovementioned period.

\section{Results}

The journal published a total of 368 papers, of which 241 (65.49\%) were original articles, and the productivity index was 2.38. Eighty-five institutions were represented by these papers. The main language is Portuguese, with 349 articles (94.84\%). There were 1574 keywords, of which 1135 (72.11\%) corresponded to DeCS or MeSH descriptors, and 102 articles (27.72\%) with all keywords being equivalent to descriptors. Study of the keywords revealed a clear community trend. The median obsolescence of the references cited by Revista de Nutrição is 11 and the Price Index is $11.69 \%$. The percentage of self-citations is 2.08 .

\section{Conclusion}

In general, the Revista de Nutrição is a publication of international level that remains within the bibliometric indicators of the health sciences journals published in Latin American countries. The journal has an excellent equivalence between keywords and health sciences descriptors (DeCS). The obsolescence of its references was somewhat high but this changed in 2006 and 2007, the last years covered by this study.

Indexing terms: Acess to information. Journal article. Bibliometrics. Bibliometric indicators. Medical subject headings.

\section{INTRODUCCIÓ N}

Un artículo científico es la suma coherente de sus componentes, un informe escrito que describe los resultados de una investigación. Desde hace unos años la mayor parte de las comunicaciones de los resultados de las investigaciones se difunden mediante revistas, por lo que los estudios bibliométricos se presentan como herramientas fundamentales para caracterizarlas y evaluarlas'.

El reconocimiento de la utilidad del análisis de las publicaciones para el estudio de la actividad investigadora y tecnológica ha llevado a la bibliometría a experimentar un gran desarrollo. Estos estudios cuantitativos permiten conocer y evaluar la producción científica de un área de conocimiento y entre sus principales aplicaciones se encuentra el área de la política científica, siendo además utilizados en los procesos de evaluación de los resultados de la actividad investigadora. En todo caso, es indispensable que los indicadores de producción, al igual que el resto de los indicadores de Ciencia y Tecnología, sean recopilados con una metodología común, generalmente aceptada, para que sus resultados puedan ser comparados y comparables.

Asimismo, la evaluación de una publicación periódica a través de indicadores bibliométricos permite obtener resultados objetivos de gran interés, así como observar las tendencias que sigue la revista. Los resultados que obtienen y las conclusiones que se deducen pueden ayudar a los responsables de la revista a mejorar la gestión de la misma, facilitando la toma 
de decisiones en aspectos relacionados, por ejemplo, con la selección de artículos².

Dada la importancia y tradición de los temas de nutrición para los países Iberoamericanos ${ }^{3,4}$ es conveniente acercarse a la realidad de esta actividad científica y de divulgación del conocimiento, por lo que este trabajo se plantea evaluar, mediante el análisis bibliométrico, la actividad científica, la producción y el consumo de información de la Revista de Nutrição, como publicación científica del área de las ciencias de la salud, en el periodo 2001 a 2007.

\section{MÉ TO D O S}

Estudio descriptivo transversal de los resultados obtenidos del análisis bibliométrico de los artículos publicados en la Revista de Nutrição desde enero de 2001 hasta diciembre de 2007, ambos inclusive.

Los datos se obtuvieron mediante la consulta de la versión electrónica de la Red SciELO [http://www.scielo.br/scielo.php?script=sci_ serial\&pid=1415-5273\&nrm=iso\& rep=\&lng=pt]. Se consideraron todas las tipologías documentales, a excepción de las Comunicaciones a Congresos.

La distribución geográfica, al igual que la filiación institucional, se determinó teniendo en cuenta el primer firmante del artículo.

Para estudiar las referencias bibliográficas se procedió al cálculo del tamaño muestral mediante la estimación de parámetros poblacionales en una población infinita $(p=$ valor esperado $=0,5$; e $=$ precisión del intervalo 0,05; Nivel de confianza $=0,95$ ). Tamaño muestral resultante igual a 385 referencias. El método de muestreo fue el aleatorio simple sin reemplazo, tomando como base el número total de referencias bibliográficas de la revista.

Indicadores estudiados: periodicidad de las publicaciones de la revista; tipología documental publicada; índice de productividad (logaritmo del número de trabajos originales publicados); distribución geográfica de la procedencia de los artículos; filiación institucional del primer firmante e Índice de Lotka; número de autores por artículo e índice de colaboración (cociente entre el número de firmas y el número de trabajos); idioma de la publicación.

- También palabras clave utilizadas y su relación con los Descriptores de Ciencias de la Salud (DeCS) o su equivalencia con los Medical Subject Headings (Mesh) del Thesaurus de la U.S. National Library of Medicine. En este aspecto se analizó la existencia de diferencias con las restantes revistas Iberoamericanas de nutrición (Nutrición Hospitalaria, Revista Chilena de Nutrición, Anales Venezolanos de Nutrición y Archivos Latinoamericanos de Nutrición).

- Estudio de las Referencias Bibliográficas (RB): tipo de publicación, año de la $R B$, revistas referidas, idioma de la RB, Índice de firmas, edad de las RB, Semiperiodo de Burton y Kebler (Mediana de la distribución del conjunto de las referencias ordenadas por antigüedad), Índice de Price (porcentaje de referencias con edad igual o menor de 5 años) y núcleo principal de Bradford (conjunto de revistas de mayor pertinencia para un área del conocimiento).

Estudio descriptivo de los indicadores fijados mediante el cálculo de las frecuencias y porcentajes de las variables indicadas, representándose las más relevantes mediante la utilización de tablas y gráficos. Las variables cuantitativas se describieron con su Media y Desviación Standard y las cualitativas con su valor absoluto y porcentaje. Se utilizó la Mediana como medida de tendencia central, y los Percentiles para determinar el punto de corte que establece diferentes grupos.

Para comparar las medias entre más de 2 grupos para una variable cuantitativa se realizó el Análisis de la Varianza (ANOVA) utilizando el método de Bonferroni. El nivel de significación aceptado fue $\alpha \leq 0,05$ (Intervalo de confianza del 95\%).

Para la introducción y análisis de los datos se usó el programa SPSS versión 15 para Windows. El control de la calidad de la 
información se efectuó a través de dobles tablas, corrigiendo mediante la consulta con los originales los errores detectados.

\section{RESULTADOS}

La estructura de la Revista de Nutrição recogida en la Red SciELO consta en 2001 y 2002 de 3 números (volúmenes 14 y 15) más un suplemento en 2001, 4 números en 2003 y 2004 (volúmenes 16 y 17) y a partir de 2005 de 6 números (volúmenes 18, 19 y 20).

- Producción científica y tipología documental: durante el periodo estudiado se publicaron en la Revista de Nutrição un total de 368 artículos. El año 2006 fue el más productivo con 72 artículos, mientras que el 2002 resultó el de menor productividad con 33 (Tabla 1). El número de originales fue de 241 (65,49\%), el índice de productividad de 2,38 y la media de 34,43 originales/año, con diferencias entre las distintas anualidades, desde un máximo de 45 en 2007 a un mínimo de 22 en 2002 (Figura 1).

- Procedencia geográfica y filiación institucional: la distribución geográfica de los autores que aparecen como primer firmante es brasileña en 359 artículos (97,55\%);la procedencia no Iberoamericana es de 4 trabajos (1,09\%), siendo todos ellos de origen francés (Tabla 2). Un único país, Brasil, ha publicado artículos todos los años estudiados.

Se identificaron 85 instituciones con trabajos publicados. Según el Índice de Lotka, su clasificación presenta tres niveles de rendimiento: pequeños productores, el Índice de Transitoriedad (un único trabajo), donde encontramos 45 centros $(52,94 \%)$; medianos productores (entre 2 y 9 trabajos) con 31 centros $(36,47 \%)$; y grandes productores (10 ó más trabajos) donde localizamos 9 centros (10,59\%) (Tabla 3).

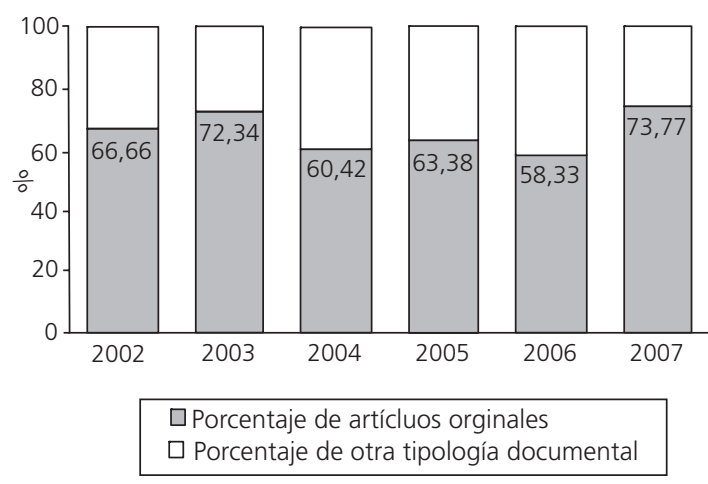

Figura 1. Porcentaje de trabajos publicados en Revista de Nutrição (2001-2007): originales y resto de trabajos.

Tabla 1. Tipología documental de los artículos publicados en Revista de Nutrição entre 2001 y 2007.

\begin{tabular}{lccccccccc}
\hline Tipo de artículo & 2001 & 2002 & 2003 & 2004 & 2005 & 2006 & 2007 & Total & $\%$ \\
\hline Original & 24 & 22 & 34 & 29 & 45 & 42 & 45 & 241 \\
Revisión & 8 & 10 & 11 & 19 & 23 & 27 & 16 & 114 & 30,98 \\
Opinión & 4 & 1 & 2 & - & 3 & 3 & - & 13 & 3,53 \\
\hline Total & 36 & 33 & 47 & 48 & 71 & 72 & 61 & 368 & 100,00 \\
\hline
\end{tabular}

Tabla 2. Distribución geográfica de los trabajos publicados en Revista de Nutrição entre 2001 y 2007.

\begin{tabular}{lccccccccc}
\hline País & 2001 & 2002 & 2003 & 2004 & 2005 & 2006 & 2007 & Total & $\%$ \\
\hline Argentina & - & - & - & 1 & - & - & - & 1 \\
Brasil & 35 & 33 & 45 & 44 & 70 & 72 & 60 & 359 & 97,55 \\
Chile & - & - & - & 1 & -- & - & - & 1 \\
España & - & - & - & 2 & - & - & 2 \\
Francia & 1 & - & 2 & - & 1 & - & - & 4 \\
Panamá & - & - & - & - & - & - & 1 & 1 \\
\hline Total & 36 & 33 & 47 & 48 & 71 & 72 & 61 & 0,27 \\
\hline
\end{tabular}


- Autoría: Se contabilizaron un total de 1322 firmantes, con un Mínimo de 1 y un Máximo de 12, siendo la Media (equivalente al Índice de cooperación) 3,59, DP=0,10 (IC95\% 3,40 - 3,78). La Mediana es igual a 3 y la Moda igual a 2. El Percentil 25 es de 2 autores y el Percentil 75 de 5 autores.

En los artículos originales se sumó un total de 953 firmantes, con Mínimo y Máximo igual que los datos anteriores y Media de 3,95, DP=0,12 (IC95\% 3,71 - 4,20). La Mediana es igual a 4 y la Moda es de 2 autores. El Percentil 25 y el Percentil 75 son de 2 y 5 autores respectivamente.

- Idioma de publicación: El idioma predominante es el portugués con 349 artículos $(94,84 \%)$, recogiéndose 15 artículos en inglés $(4,08 \%)$ y 4 en español $(1,09 \%)$.

- Palabras Clave: De los 368 artículos estudiados, 7 (1,90\%) no presentaban Palabras Clave $(\mathrm{PC})$; sin embargo, uno de los trabajos presentó 11 PC. En total se contabilizaron 1574 PC, de las cuales $1135(72,11 \%)$ se correspondían con los términos DeCS o MeSH; 102 artículos $(27,72 \%)$ se relacionaron con PC equivalentes a Descriptores. Las dos PC más usadas fueron dieta y estado nutricional (Tabla 4).

La diferencia de medias de utilización de PC equivalentes a Descriptores fue en todos los casos favorable a la Revista de Nutrição al compararse con las revistas: Nutrición Hospitalaria, Revista Chilena de Nutrición, Anales Venezolanos de Nutrición y Archivos Latinoamericanos de Nutrición; $p<0,001$.

- Referencias: El número total de referencias es de $11329 ; 340$ de ellas (3,00\%) están escritas en formato electrónico, lo que representa una relación de 33:1. Presenta Mínimo de 0 y Máximo de 96 referencias. La Media de referencias por artículo es de 30,79, DP=0,71 (IC95\% 29,38 - 32,19), la Mediana igual a 29 y la Moda es de 30 autores. El Percentil 25 y el Percentil 75 son de 22 y 36 autores, respectivamente.

La muestra estudiada (385 referencias) mostró la tipología documental citada: artículos de revistas 67,79\% (IC95\% 63,12 - 72,46) (Tabla 5).
Tabla 3. Instituciones con más de 10 trabajos en Revista de Nutrição entre 2001 y 2007.

\begin{tabular}{lcc}
\hline País & Total & $\%$ \\
\hline Universidade de Sao Paulo & 53 & 14,40 \\
Universidade Federal de Visçosa & 38 & 10,33 \\
Universidade Federal do São Paulo & 23 & 6,25 \\
Universidade Federal Santa Caterina & 22 & 5,98 \\
Universidade Federal do Rio Janeiro & 19 & 5,16 \\
Universidade Estadual Paulista & 15 & 4,08 \\
Universidade Federal de Pernambuco & 14 & 3,80 \\
Universidade Estadual de Campinas & 14 & 3,80 \\
Universidade Federal de Bahia & 13 & 3,53 \\
\hline
\end{tabular}

Tabla 4. Palabras clave utilizadas más de 10 veces en los artículos publicados en Revista de Nutrição (enero 2001 a diciembre 2007) y su equivalencia con los Medical Subject Headings o Descriptores de Ciencias de la Salud.

\begin{tabular}{lcccc}
\hline Palabra clave & $\mathrm{f}_{0}$ & $\%$ & MeSH & DeCS \\
\hline Estado nutricional & 33 & 1,38 & $\mathrm{Si}$ & $\mathrm{Si}$ \\
Dieta & 33 & 1,38 & $\mathrm{Si}$ & $\mathrm{Si}$ \\
Obesidad & 31 & 1,30 & $\mathrm{Si}$ & $\mathrm{Si}$ \\
Nutrición & 30 & 1,26 & $\mathrm{No}$ & $\mathrm{Si}$ \\
Consumo de alimentos $^{*}$ & 28 & 1,17 & $\mathrm{No}$ & $\mathrm{Si}$ \\
Antropometría & 23 & 0,96 & $\mathrm{Si}$ & $\mathrm{Si}$ \\
Niño & 22 & 0,92 & $\mathrm{Si}$ & $\mathrm{Si}$ \\
Adolescente & 20 & 0,84 & $\mathrm{Si}$ & $\mathrm{Si}$ \\
Evaluación nutricional & 19 & 0,80 & $\mathrm{Si}$ & $\mathrm{Si}$ \\
Hábitos alimenticios & 16 & 0,67 & $\mathrm{Si}$ & $\mathrm{Si}$ \\
Ratones & 13 & 0,54 & $\mathrm{Si}$ & $\mathrm{Si}$ \\
Nutricionista & 12 & 0,50 & $\mathrm{No}$ & $\mathrm{Si}$ \\
Alimentación & 11 & 0,46 & No & $\mathrm{Si}$ \\
Amamantamiento* & 11 & 0,46 & $\mathrm{Si}$ & $\mathrm{Si}$ \\
Educación nutricional & 11 & 0,46 & No & $\mathrm{Si}$ \\
\hline
\end{tabular}

* No es un MeSH o DeSC pero es un sinónimo aceptado.

Tabla 5. Distribución porcentual de la tipología documental referida en Revista de Nutrição en el periodo 2001 a 2007.

\begin{tabular}{lrr}
\hline Tipo documental & $\%$ & \multicolumn{1}{c}{ IC $95 \%$} \\
\hline Artículo original & 67,79 & $63,12-72,46$ \\
Memoria e informe & 14,55 & $11,02-18,07$ \\
Libro & 8,57 & $5,78-11,37$ \\
Capítulo de libro & 4,42 & $2,36-6,47$ \\
Tesis & 1,56 & $0,32-2,80$ \\
Monografía en Internet & 1,56 & $0,32-2,80$ \\
Programa informático & 0,78 & $0,00-1,66$ \\
En prensa & 0,52 & $0,00-1,24$ \\
Página Web & 0,26 & $0,00-0,77$ \\
\hline
\end{tabular}


El número de autores por referencia presenta una Mediana de 3 y Moda de 1 , advirtiéndose un 11,43\% (IC95\% 8,25 - 14,61) de artículos con más de 6 firmantes.

El idioma de las referencias es el inglés en un $65,19 \%$ (IC95\% 60,44 - 69,95), seguido del portugués con el 28,31\% (IC95\% 23,81 - 32,81), el español con un 4,16\% (IC95\% 2,16 - 6,15), el francés con el 1,82\% (IC95\% 0,48 - 3,15) y el sueco con el 0,26\% (IC95\% 0,00 - 0,77).

La obsolescencia de las referencias citadas en la Revista de Nutrição, medida por la Mediana
(Semiperiodo de Burton y Kebler), es de 11 y el Índice de Price es del 11,69\%. En el Figura 2 se puede observar que, si bien en todos los años existen algunas referencias con edades que se sitúan fuera de los límites del gráfico de caja, referencias representadas por (o) puntos outliner $y\left({ }^{*}\right)$ extremos, se puede constatar que la mediana de cada uno de los años (línea interior de la caja) va disminuyendo progresivamente, situándose en los años 2006 y 2007 por debajo del valor medio.

El estudio de la dispersión de la literatura científica citada, determina la concentración de

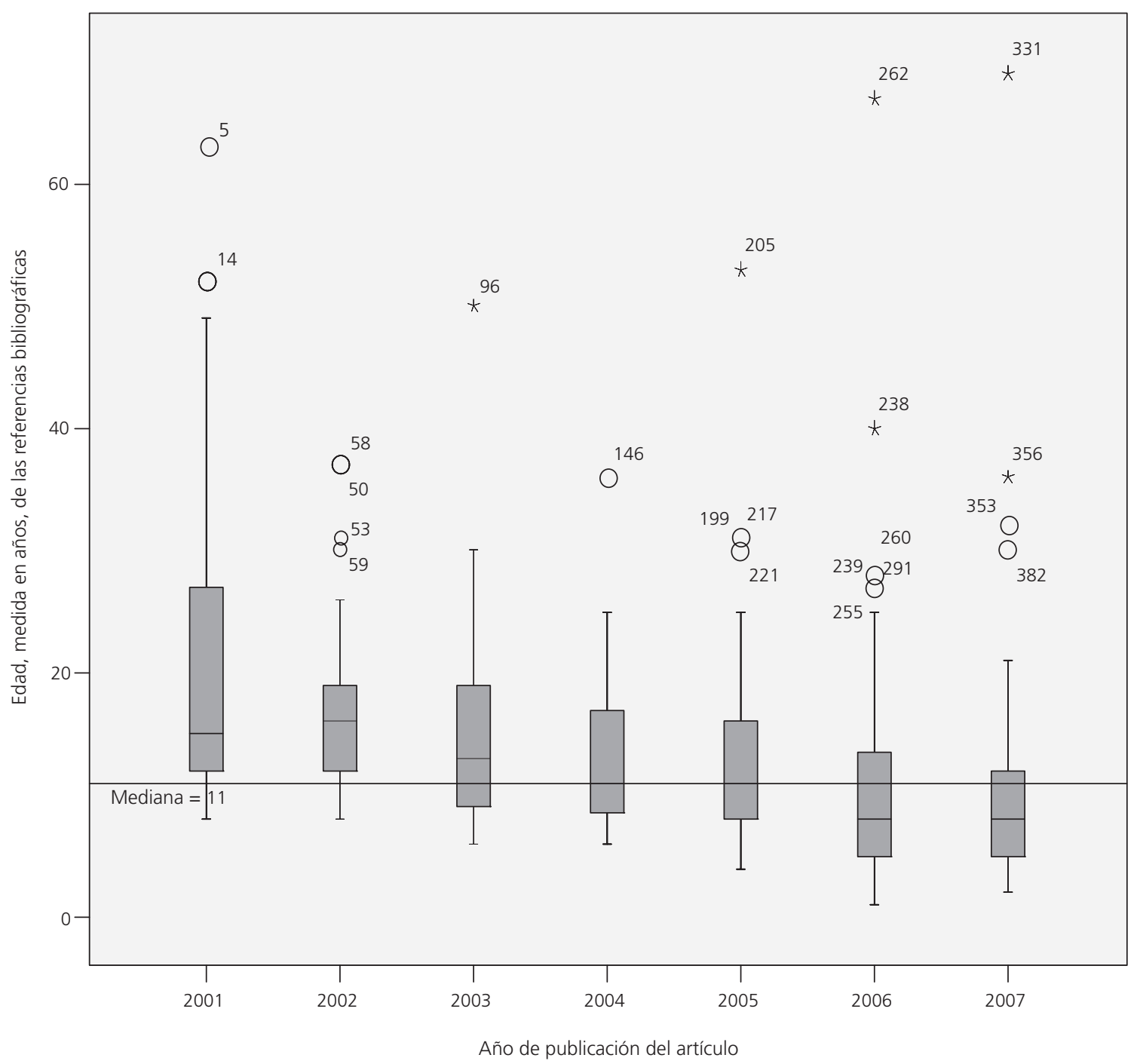

Figura 2. Edad de las referencias bibliográficas contenidas en los artículos publicados en Revista de Nutrição. 
Tabla 6. Revistas referenciadas en Revista de Nutrição (2001 a 2007) pertenecientes al núcleo principal de Bradford.

\begin{tabular}{lccc}
\hline Título Revista & $\%$ & IC 95\% & F.I. $^{*}$ \\
\hline Am J Clin Nutr & 7,01 & $4,46-9,56$ & 6,603 \\
J Nutr & 3,38 & $1,57-5,18$ & 3,3771 \\
Rev Saude Publica & 2,60 & $1,01-4,19$ & - \\
Rev Nutr & 2,08 & $0,65-3,50$ & - \\
J Am Diet Assoc & 1,82 & $0,48-3,15$ & 3,011 \\
Br J Nutr & 1,56 & $0,32-2,80$ & 2,339 \\
Arch Latinoam Nutr & 1,30 & $0,17-2,43$ & 0,266 \\
Cad Saúde Pública & 1,04 & $0,03-2,05$ & - \\
J Agric Food Chem & 1,04 & $0,03-2,05$ & 2,532 \\
J Appl Physiol & 1,04 & $0,03-2,05$ & 3,632 \\
\hline
\end{tabular}

* F.I.: factor de impacto, datos obtenidos de 2007 JCR Science Edition Database, de la ISI Web of Knowledge, Thomson Reuters ${ }^{\odot}$.

un porcentaje aproximado de documentos en un número diferente de revistas: núcleo principal, 10 revistas $(33,72 \%)$ que recogen 88 referencias (Tabla 6); núcleo 2 con 33,33\% de referencias se recoge en 56 revistas y núcleo 3 con un 32,95\% de referencias que se recogen en 86 revistas. El porcentaje de autocitas es de 2,08\% (IC95\% $0,65-3,50)$.

\section{DISCUSIÓN}

Es primordial señalar la disponibilidad de los datos estudiados en el texto completo de la producción científica publicada en formato electrónico en la Red SciELO.

Revista de Nutrição es una publicación que, como muestran los datos de producción, ha crecido en los últimos años. El enfoque iberoamericano de esta revista es incuestionable. Pero el equipo editorial de la revista debería debatir la escasez de artículos con otra filiación . La orientación de la revista es claramente comunitaria, basta ver las principales Palabras Clave utilizadas para comprobarlo.

La producción científica calculada, el número de autores y el índice de cooperación presentan datos similares a otra revistas sobre ciencias de la salud del área iberoamericana ${ }^{2,5}$, aunque debe reconocerse que la proporción de artículos originales supera los valores habituales ${ }^{1,6,7}$.
El predominio del idioma nacional es una constante en las revistas iberoamericanas sobre nutrición. Los autores con mayor capacidad idiomática tienden a publicar en revistas de habla anglófona una vez realizado el esfuerzo de escribir el artículo en inglés. Además éstos pueden ser amparados por su propia institución, que impulsa y recomienda el envío a revistas de alto impacto aunque ello suponga tener que pagar por la posterior recuperación del artículo o incluso por su revisión, jtodo por el "impacto"!8.

El resultado más destacable y muy superior a lo observado hasta el momento en revistas iberoamericanas sobre nutrición e incluso del campo de las ciencias de la salud es el porcentaje de Palabras Clave que coinciden con Descriptores de Ciencias de la Salud o con Medical Subject Headings. Este dato denota, ya sea un buen conocimiento de los Descriptores por parte de los autores, o un cuidado del equipo editorial en su correcta utilización ${ }^{9,10}$.

Las tasas anuales de referencias por publicación son acordes a resultados de estudios previos $^{11-13}$, teniendo en cuenta siempre las limitaciones a los autores impuestas por las normas a la hora de posibles comparaciones.

La tipología documental referida por los autores para respaldar sus trabajos es también similar a lo observado en estudios previos. En este sentido, citar artículos de revistas científicas es un hecho usual, acrecentado por la facilidad de localización motivada por las bases de datos bibliográficas (MedLine, Lilacs, etc.). Las revistas más referidas coinciden con publicaciones sobre las ciencias de la nutrición. Asimismo, el hecho de que se mencionen artículos publicados en revistas de alto impacto ya ha sido estudiado ${ }^{14}$, es por tanto esperable que este grupo de revistas concentren el mayor número de citas y que en consecuencia se sitúen en el núcleo principal de Bradford, reafirmando la importancia que los autores relacionados con el campo de la nutrición dan a las publicaciones periódicas de mayor consulta. 
Revista de Nutrição muestra porcentajes de autocitas por debajo de los estudios bibliométricos ya citados en este artículo. Sin embargo, resulta lógico que los autores que publican en una revista la citen, ya que divulga temas relacionados con su área de conocimiento ${ }^{15}$. En todo caso, siempre hay que tener en cuenta que mejorar la visibilidad científica de los autores, complacer a superiores, a editores o revisores e incluir citas de prestigio con la intención de incrementar el peso de las evidencias son otros motivos que influyen en la selección de la bibliografía ${ }^{16,17}$. Nada nuevo que no se sepa y ya haya sido comprobado ${ }^{18,19}$.

El análisis de la obsolescencia, medido tanto por la Mediana como por el Índice de Price, muestra resultados que penalizan los indicadores de actualidad de la revista. Estos datos se sitúan en el campo de las ciencias de la salud en una mediana igual a 7 años y un índice de Price en torno al 33\%6,12,13. Esta situación presenta una tendencia claramente favorable, descendente, que se aprecia patentemente en el Figura 2.

\section{CONCLUSIONES}

Como se ha constatado, Revista de Nutrição, publicación de ámbito internacional, presenta unos indicadores bibliométricos similares a las revistas punteras de las ciencias de la salud publicadas en países iberoamericanos y totalmente equiparables en los dos últimos años estudiados. Hay que destacar los excelentes resultados en la equivalencia de las Palabras Clave con los Descriptores de Ciencias de la Salud. Al mismo tiempo, presenta un buen porcentaje de artículos originales en relación a la tipología documental.

\section{A GRADECIMIENTOS}

Este artículo forma parte del trabajo para la obtención de la Tesis Doctoral por compendio de publicaciones de Don Vicente Tomás-Casterá. Los autores quieren agradecer el esfuerzo editorial y las sugerencias formuladas por los revisores, ya que han contribuido a mejorar la calidad del artículo.

\section{REFERENCIAS}

1. Pérez Andrés C, Estrada Lorenzo JM, Villar Álvarez F, Rebollo Rodríguez MJ. Estudio bibliométrico de los artículos originales de la Revista Española de Salud Pública (1991-2000), parte primera: indicadores generales. Rev Esp Salud Pública. 2002; 76(6): 659-72.

2. Granda-Orive Jl, García Río F, Gutiérrez Jiménez T, Jiménez Ruiz CA, Solano Reina S, Sáez Valls R. Análisis y evolución de los indicadores bibliométricos de producción y consumo del área de tabaquismo a través de Archivos de Bronconeumología (período 1970-2000): comparación con otras áreas neumológicas. Arch Bronconeumol. 2002; 38(11):523-9.

3. Díaz Mújica D. Análisis bibliométrico de la revista Archivos Latinoamericanos de Nutrición. An Venez Nutr. 2007; 20(1):22-9.

4. de Lorenzo-Cáceres A, Otero Puime A. Publicaciones sobre evaluación de la atención primaria en España tras veinte años de reforma (1984-2004): análisis temático y bibliométrico. Rev Esp Salud Pública. 2007; 81(2):131-45.

5. Pérez Andrés C, Estrada Lorenzo JM, Villar Álvarez F, Rebollo Rodríguez MJ. Estudio bibliométrico de los artículos originales de la revista española de la salud pública (1991-2000). Parte primera: indicadores generales. Rev Esp Salud Pública. 2002; 76(6):659-72.

6. Valera Garrido JF, Gala Sánchez F. Análisis bibliométrico de la productividad en la revista Mapfre Medicina. Mapfre Med. 2001; 12(3):157-67.

7. González de Dios J. Evaluación de la calidad de la evidencia científica en Anales Españoles de Pediatría. An Esp Pediatr. 2001; 54(6):605.

8. Casterá VT, Sanz-Valero J, Juan-Quilis V, WandenBerghe C, Culebras JM, García de Lorenzo A, et al. Estudio bibliométrico de la revista Nutrición Hospitalaria en el periodo 2001 a 2005: Parte I, análisis de la producción científica. Nutr Hosp. 2008; 23(5):469-76.

9. Granda-Orive Jl, García Río F, Callol Sánchez L. Importancia de las Palabras Clave en las búsquedas bibliográficas. Rev Esp Salud Publica. 2003; 77(6): 765-7.

10. Granda-Orive Jl, García Río F, Roig Vázquez F, Escobar Sacristán J, Gutierrez Jiménez T, Callol Sánchez L. Las Palabras Clave como herramientas imprescindibles en las búsquedas bibliográficas: análisis de las áreas del sistema respiratorio a través de Arch Bronconeumol. Arch Bronconeumol. 2005; 41(2);78-83. 
11. Miralles J, Ramos JM, Ballester R, Belinchón I, Sevilla A, Moragón M. Estudio bibliométrico de la revista Actas Dermo-Sifiliográficas (1984-2003). Análisis de las referencias bibliográficas. Actas Dermosifiliogr. 2005; 96(9):563-71.

12. Villar Álvarez F, Estrada LJM, Pérez AC, Rebollo RMJ. Estudio bibliométrico de los artículos originales de la Revista Española de Salud Pública (1991-2000) Parte tercera: análisis de las referencias bibliográficas. Rev Esp Salud Publica. 2007; 81(3): 247-59.

13. Casterá VT, Sanz-Valero J, Juan-Quilis V, WandenBerghe C, Culebras JM, García de Lorenzo A, et al. Estudio bibliométrico de la revista Nutrición Hospitalaria en el periodo 2001 a 2005: Parte II, análisis de consumo; las referencias bibliográficas. Nutr Hosp. 2008; 23(6):541-6.

14. Callaham M, Wears RL, Weber E. Journal prestige, publication bias, and other characteristics associated with citation of published studies in peer-reviewed journals. JAMA. 2002; 287(1): 2847-50.
15. Iglesias Vázquez E, Culebras JM, García de Lorenzo A. Evaluación de Nutrición Hospitalaria (III): tiempos de demora, materias y citación. Nutr Hosp. 2002; 17(1):34-42.

16. Castiel LD, Sanz-Valero J. Entre fetichismo e sobrevivência: o artigo cientifico é uma mercadoria acadêmica? Cad Saúde Pública. 2007; 23(12): 3041-50.

17. Aleixandre Benavent R, Valderrama Zurián JC, Miguel-Dasit A, Granda Orive JL. El factor de impacto de Revista Iberoamericana de Micología. Rev Iberoamericana Micología. 2004; 21(1):161-7.

18. Kiserud T, Nylenna M. Citations and reference lists: for whom and for what purpose? Ultrasound Obst Gynecol. 2003; 22(2):105-7.

19. Culebras Fernández JM, García de Lorenzo A Wanden-Berghe C, Castiel LD, Sanz-Valero J. ¡Cuidado!, sus referencias bibliográficas pueden ser estudiadas. Nutr Hosp. 2008; 23(2):85-8.

Recebido el: 9/12/2008

Versión final re-apresentada el: 22/7/2009

Aprobado el: 4/5/2010 
